\title{
Clinical and Radiological Characterisation of Patients with Mucopolysaccharidosis in a Genetic Clinic
}

Journal of Inborn Errors of Metabolism \& Screening 2021, Volume 9: e20200008 DOI: https://doi.org/10.1590/2326-4594JIEMS-2020-0008

\author{
Pallavelangini Swetha' ${ }^{1}$, Inusha Panigrahi' ${ }^{1}$, Akshay Saxena ${ }^{2}$, \\ Anupriya Kaur ${ }^{1}$ and Rozy Thakur ${ }^{1}$
}

\begin{abstract}
The mucopolysaccharidoses (MPS) are a relatively uncommon group of inherited metabolic disorders. MPSs should be suspected in a child with coarse facies, organomegaly, recurrent respiratory tract infections, developmental delay, and hernias. Early diagnosis and treatment can greatly improve the quality of life in these children. In this study we studied 46 MPS patients diagnosed on enzyme and/or DNA testing and we found that the MPS II was the most common type followed by MPS I and MPS IVA. While the mean age of onset of symptoms was 12 months, the mean age at diagnosis was 4.5 years, a significant delay. One of major presenting features was recurrent respiratory problems, more prevalent in MPS II cases. Many patients also had short stature and contractures. Increasing awareness among physicians is of paramount importance for the early diagnosis and optimal treatment and prevention by prenatal testing and counselling.
\end{abstract}

Keywords: Lysosomal disorders, mucopolysaccharidoses, glycosaminoglycans, Enzyme assay, coarse facies, organomegaly, short stature and dysostosis.

\section{Introduction}

The mucopolysaccharidoses (MPS) constitute one of the main groups of the lysosomal storage disorders and include several disorders with overlapping features and a combined incidence of 1 in 25,000 live births. The most common are MPS I, MPS II, MPS III $(B>A>C)$ and MPS IV $(A>B)$, with incidences and relative frequencies of that vary from region to region [1-4]. MPS II is inherited as an X-linked recessive trait while all the others have autosomal recessive inheritance. MPS III is divided into four subtypes-A, B, C, and D; and MPD IV is of 2 subtypes IV-A and IV-B (Table 1). The population prevalence in India is not known since no universal newborn screening is available for lysosomal storage disorders (LSDs) or MPS. However, the mutation spectrum of MPS I and MPS II patients has been described in a previous study [3].

MPS are a group of disorders which occur secondary to deficient activity of the lysosomal enzymes which are required to degrade glycosaminoglycans (GAGs). The major GAGs are chondroitin-4-sulphate, chondroitin-6-sulphate, heparain sulphate, dermatan sulphate, keratan sulphate and hyaluronan [6]. GAG accumulation causes damage to various tissues and organs which leads to skeletal deformities, short stature, joint rigidity, myelopathy (due to accumulation of keratan, chondroitin and dermatan sulphates), intellectual disability, behavioural abnormalities (due to accumulation of heparan sulphate), coarse facies, organomegaly, corneal clouding, hearing loss, recurrent respiratory tract infections, cardiac compromise and hydrops fetalis as shown in Table 1. In this report, we present the clinical and radiological features of different MPS followed in the Genetic clinic.

\section{Material and Methods}

Postnatal and antenatal cases, including patients aged 1-18 years old attending the Genetic Metabolic Unit, PGIMER, in Chandigarh, India, during a period of 2 years (2018-2019) were included in the analysis. Patients who were clinically diagnosed

\footnotetext{
${ }^{1}$ Post Graduate Institute of Medical Education \& Research, Pediatrics, Chandigarh India.

${ }^{2}$ Post Graduate Institute of Medical Education \& Research, Radiodiagnosis, Chandigarh, India.
}

Received June 13, 2020, and in revised form January 05, 2021. Accepted for publication January 22, 2021.

\section{Corresponding Author:}

Pallavelangini Swetha, Post Graduate Institute of Medical Education \& Research, Pediatrics, Chandigarh, India.

Email: pallavelanginiswetha@gmail.com 
Table 1. Types of mucopolysaccharidoses (MPS) and clinical features [2]

\begin{tabular}{|c|c|c|c|}
\hline MPS Type & Eponym(Gene) & Clinical features & Defective enzyme \\
\hline I-H & $\begin{array}{l}\text { Hurler } \\
\text { (IDUA) }\end{array}$ & $\begin{array}{c}\text { Intellectual disability, hepatosplenomegaly, optic atrophy, } \\
\text { corneal clouding, death usually before } 14 \text { years; severe } \\
\text { cases-foetal hydrops. }\end{array}$ & a-L Iduronidase \\
\hline I-S & $\begin{array}{l}\text { Scheie } \\
\text { (IDUA) }\end{array}$ & $\begin{array}{l}\text { Stiff joints, normal intelligence, corneal clouding, } \\
\text { aortic valve disease, survive to adulthood. }\end{array}$ & a-L Iduronidase \\
\hline I-HS & $\begin{array}{l}\text { Hurler-Scheie } \\
\quad \text { (IDUA) }\end{array}$ & $\begin{array}{l}\text { Phenotype intermediate } \\
\text { between I-H and I-S }\end{array}$ & a-L Iduronidase \\
\hline II & Hunter(IDS) & $\begin{array}{l}\text { Course similar to I-H but clear corneas. Mild course } \\
\text { less pronounced features, survival to adulthood with } \\
\text { mild or without mental retardation. }\end{array}$ & Iduronate sulfatase \\
\hline III-A & $\begin{array}{l}\text { Sanfilippo-A } \\
\text { (SGSH) }\end{array}$ & & Heparan-S-sulfamidase \\
\hline III-B & $\begin{array}{l}\text { Sanfilippo- B } \\
\text { (NAGLU) }\end{array}$ & Behavioural problems, mild dysmorphism, sleep & N-Acetyl alpha D glucosaminidase \\
\hline III-C & $\begin{array}{l}\text { Sanfilippo-C } \\
\text { (HGSNAT) }\end{array}$ & progressive dementia, survival to adulthood possible. & $\begin{array}{l}\text { Heparan glucosaminide } \\
\text { N-Acetyltransferase }\end{array}$ \\
\hline III-D & $\begin{array}{l}\text { Sanfilippo-D } \\
\text { (GNS) }\end{array}$ & & N- Acetylglucosamine 6 sulfatase \\
\hline IV-A & $\begin{array}{l}\text { Morquio A } \\
\text { (GALNS) }\end{array}$ & $\begin{array}{l}\text { Short trunk dwarfism, characteristic bone dysplasia, } \\
\text { fine corneal opacities, final height below } 125 \mathrm{~cm} \text {. }\end{array}$ & N-Acetyl-galactosamine-6-sulfatase \\
\hline IV-B & $\begin{array}{l}\text { Morquio B } \\
\text { (GLB1) }\end{array}$ & Same as IV-A but milder; adult height is over $120 \mathrm{~cm}$. & Beta galactosidase \\
\hline VI & $\begin{array}{l}\text { Maroteaux-Lamy } \\
\text { (ARSB) }\end{array}$ & $\begin{array}{l}\text { Hurler like phenotype with marked corneal clouding } \\
\text { but normal intelligence, dysmorphism, carpal tunnel } \\
\text { syndrome, varying phenotype in different families. }\end{array}$ & N-Acetyl galactosamine 4 sulfatase \\
\hline VII & Sly (GUSB) & $\begin{array}{l}\text { Varying from foetal hydrops to mild dysmorphism, } \\
\text { dense inclusions in granulocytes. }\end{array}$ & Beta glucoronidase \\
\hline $\mathbf{I X}$ & Hyaluronidase deficiency(HYAL1) & $\begin{array}{l}\text { Periarticular masses, short stature, bifid uvula, } \\
\text { joint effusion, otitis media. }\end{array}$ & Hyaluronidase 1 \\
\hline
\end{tabular}

as MPS-old and new patients; were further investigated biochemically by evaluation of the excretion of urinary GAGs and enzyme analysis in blood. The cases in which MPS was confirmed were enrolled. Complete clinical features were recorded in a predesigned form. Skeletal survey of all possible cases was collected for interpretation of radiological changes. WHO growth charts were used for identifying short stature (height $<5^{\text {th }}$ centile). Standard spectrophotometric method was used for measurement of urine GAGs. In clinically suspected cases enzyme analysis was done in blood leukocytes (heparinised sample) using 4-MU specific substrates. Beta-galactosidase was used as a reference (control) enzyme for most MPS case studies. In antenatal cases, amniotic fluid enzyme testing for MPS I, II, IV, and GM1 gangliosidosis was performed in foetal hydrops cases. The study is as per recommendations/the ethical principles in Declaration of Helsinki. Written informed consent was obtained from patients for enrolment and genetic testing; and the study was approved by institutional ethics committee (IEC).

\section{Results}

A total of 46 confirmed MPS patients were enrolled in the study. The type of MPS was determined on enzyme testing. Level of urine GAGs in the patients ranged from $8-82 \mathrm{mg} / \mathrm{mmol}$ creatinine. Among the various subtypes MPS-II was the most common (14/46; 30\%) followed by MPS-I (13/46; $28 \%)$ and MPSIV (11/46; 24\% IVA-7, IV B-4), MPS VI (6/46; 13\%) and MPS IIIC $(2 / 46 ; 4 \%)$. In the same period of 2 years, 2 antenatal cases of hydrops were identified as MPS I and MPS IV of 6 cases of hydrops tested by lysosomal enzyme analysis on amniotic fluid.

Among the children with MPS presenting in postnatal period, males were $73.9 \%(34 / 46)$ and females were $26 \%(12 / 46)$ with a male to female ratio of 2.8: 1 . Family history was positive in $28 \%$ of the cases. Median age of onset of symptoms was 12 months with interquartile range from $8-31.5$ months. Median age of diagnosis was 4.5 years. $39 \%$ of the children had weight $<5^{\text {th }}$ centile and $78 \%$ of them had height $<5^{\text {th }}$ centile according to World Health Organisation (WHO) growth charts.

The most common early clinical characteristics observed in MPS cases, were coarse facies (42/46; 92\%), developmental delay (34/46; 75\%), short stature $(31 / 46 ; 68 \%)$ followed by recurrent upper and lower respiratory tract infections (rhinitis and pneumonia)(19/46; 41\%). Seizures, behavioural abnormalities, abdominal distension and contractures were the presenting complaints in $16 \%$ of the children.[4] Facial profile of some patients with different types of MPS are shown in Figure 1. 
Clinical symptoms in various types of MPS and findings on specialist evaluation have been attached in figures 2 and 3 respectively.

The clinical manifestations in various types of MPS are described as follows. MPS-I children at presentation had coarse facies in $100 \%$, developmental delay and short stature with joint contractures were seen in more than $50 \%$. A quarter of them also had visual impairment. Skeletal involvement with delayed dentition, genu valgum and pectus carinatum were seen in some. Few also had significant valvulopathy and cardiomyopathy. MPS-II children also had coarse facies at presentation (100\%), followed by developmental delay and joint contractures seen in $86 \%$ each. Short stature was seen in $79 \%$ of the cases followed by recurrent respiratory tract infections in $71 \%$ of them (highest among all the subgroups). One child had autoimmune haemolytic anaemia (AIHA) and immune thrombocytopenia with history of intracapsular bleed requiring treatment with steroids and on imaging was found to have internal carotid artery stenosis. Immune thrombocytopenia was due to Epstein Barr viral infection which further complicated the presentation. [5] Skin manifestations were present in $57 \%$ of the cases in form of Mongolion spots, callosities over joints. Seizures and behavioural abnormalities were also present in significant proportion; one child had attention deficit hyperactivity disorder (ADHD) requiring treatment with risperidone. Valvulopathy was found in $60 \%$ in form of aortic regurgitation (AR), mitral regurgitation (MR) and mitral valve prolapse (MVP) along with dilated cardiomyopathy and left ventricular hypertrophy. Intellectual disability was found in $100 \%$. Adeno-tonsillitis with snoring and open mouth breathing was also present. MPS-III was the least common subtype. We had 2 children and both of them had coarse facies and intellectual disability. One of them was diagnosed as a case of autism spectrum disorder and was started on behavioural therapy. MPS-IVA children had mild coarse facies and motor delay in majority. They had short stature $\left(<5^{\text {th }}\right.$ centile) and other skeletal manifestations - kyphoscoliosis, genu valgum, chest wall abnormalities. Many of them also had history of upper recurrent respiratory tract infections. Joint contractures were not seen in this subgroup and none of them had vision or hearing impairment. MPS-VI children had coarse facies, developmental delay, and joint contractures in majority. Significant proportion also had valvulopathy.

Skeletal survey was also done and the most common findings were oar/paddle shaped ribs (43\%), J-shaped sella (41\%), poorly developed acetabulum (41\%), hip dysplasia (35\%), proximal pointed metacarpals (33\%) and anterior hypoplasia of lumbar vertebrae (32\%) followed by kyphoscoliosis (Figure 4, Figure 5). Other uncommon findings found were beaking of vertebral bodies with anterior hypoplasia of lumbar vertebrae, rounded iliac wings with inferior tapering, long femoral neck, short broad metacarpals with V-shaped hypoplastic distal ulna and radius. In some children brain neuroimaging was done and they showed common findings - cerebral atrophy, white matter hyperintensities suggestive of leukodystrophy, enlarged Virchow

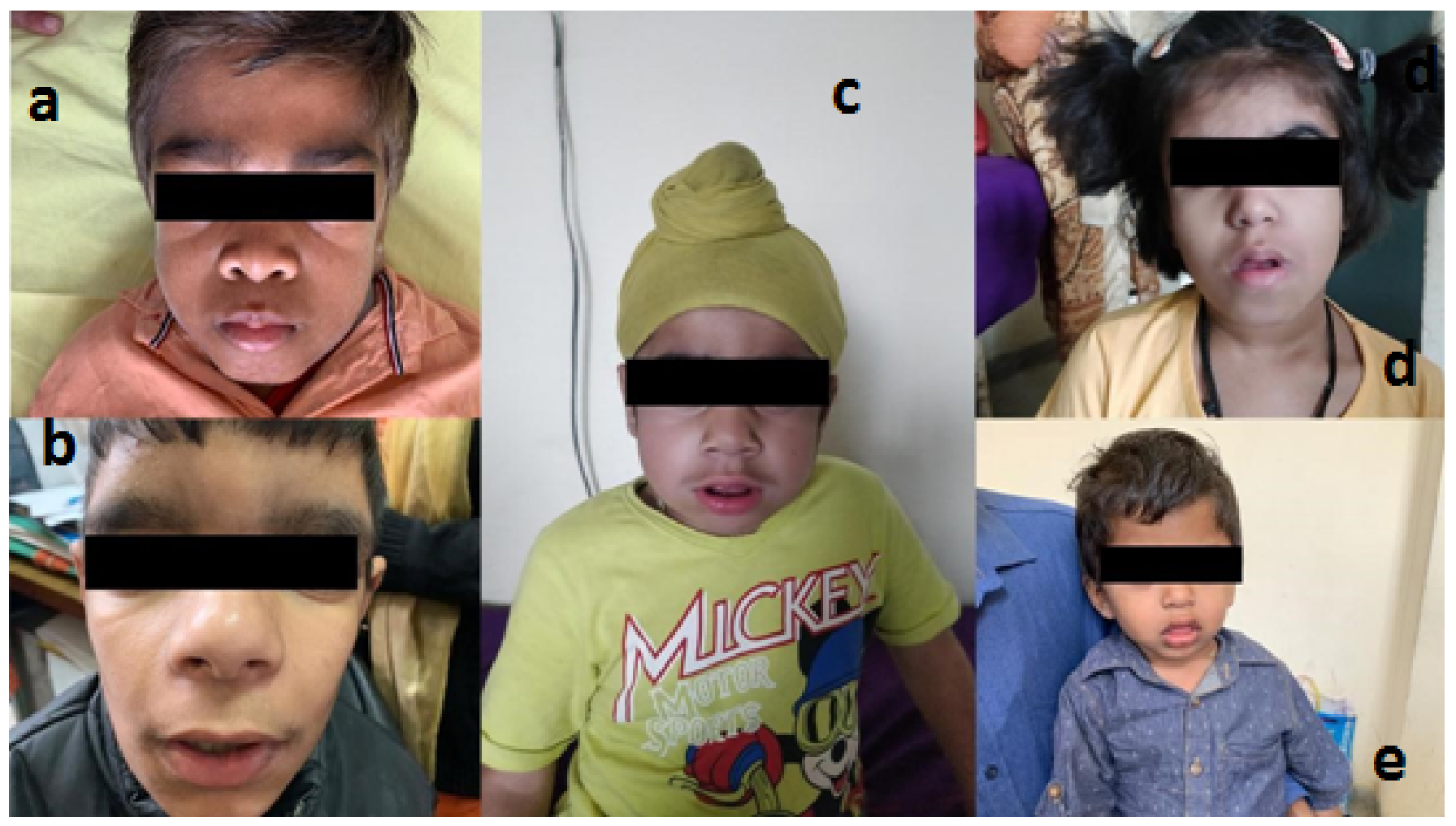

Figure 1. Facial profile of various MPS showing coarse facies, thick lips and open mouth: MPS II (1a, 1b, 1e); MPS I (1c); MPS IIIA (1d) (with consent-). 


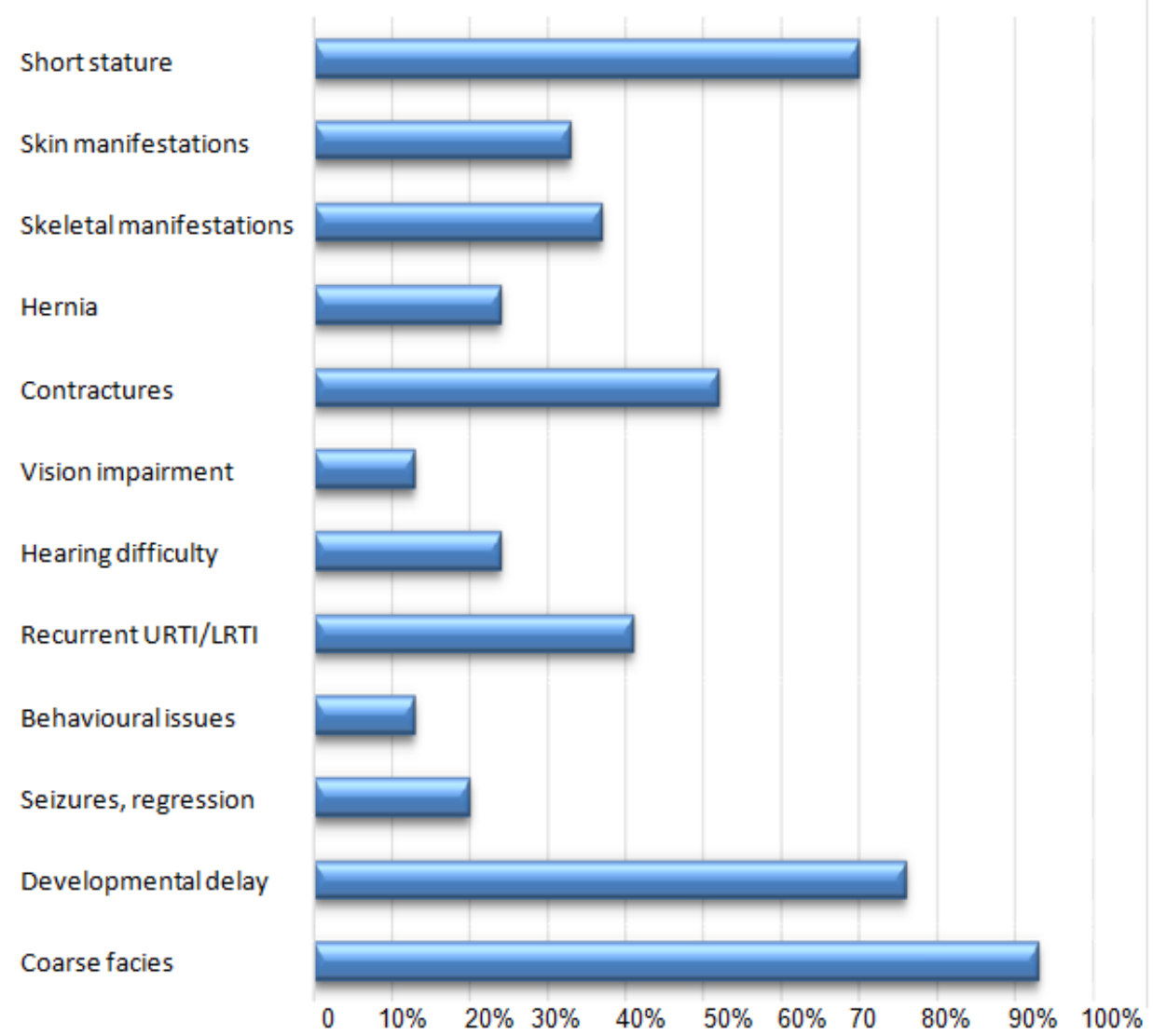

Figure 2. Clinical symptoms in various types of MPS in present study.

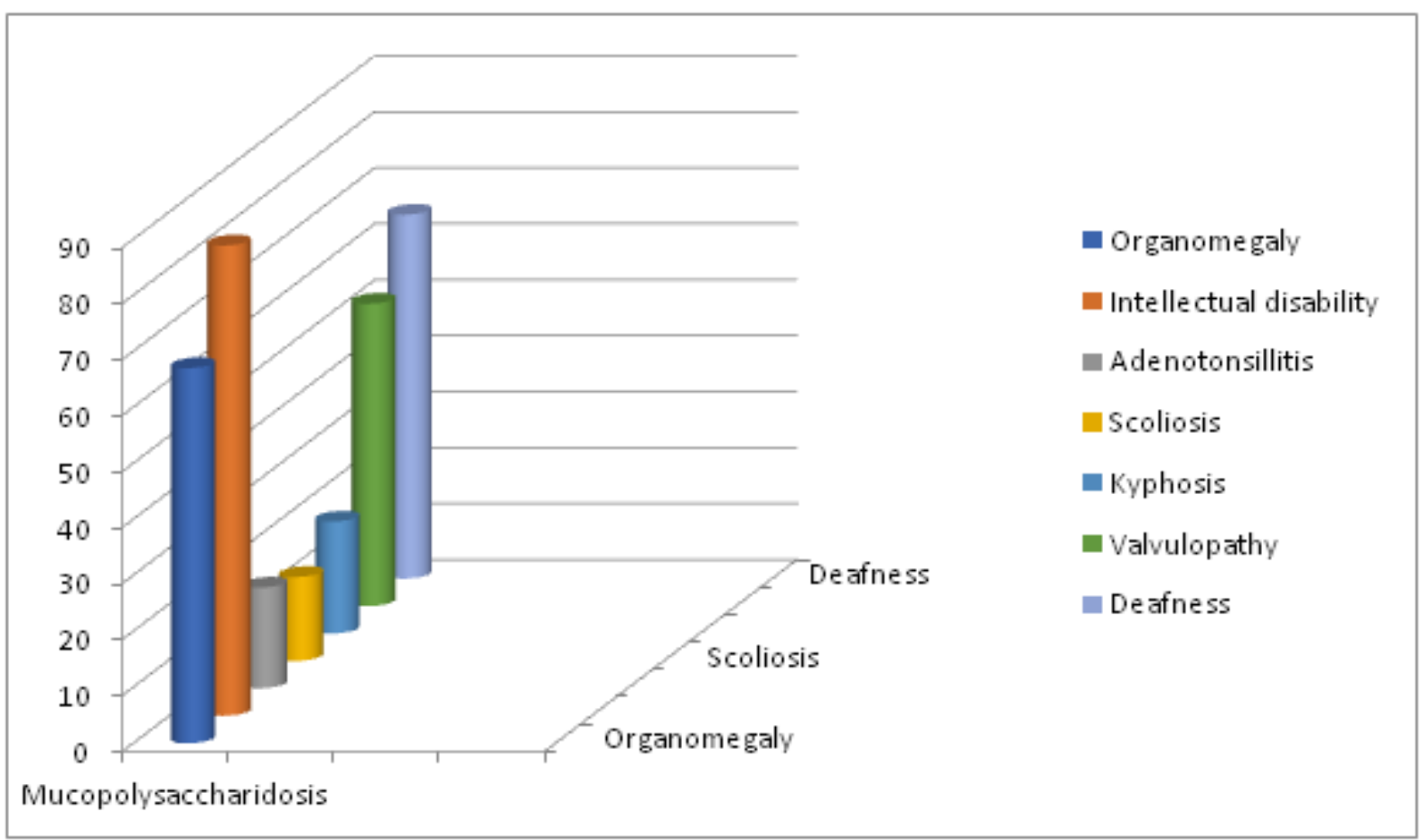

Figure 3. Results of specialist evaluation in MPS patients. Organomegaly includes hepatomegaly and splenomegaly. Valvulopathy includes aortic regurgitation (AR), mitral regurgitation (MR) and mitral valve prolapse (MVP). 


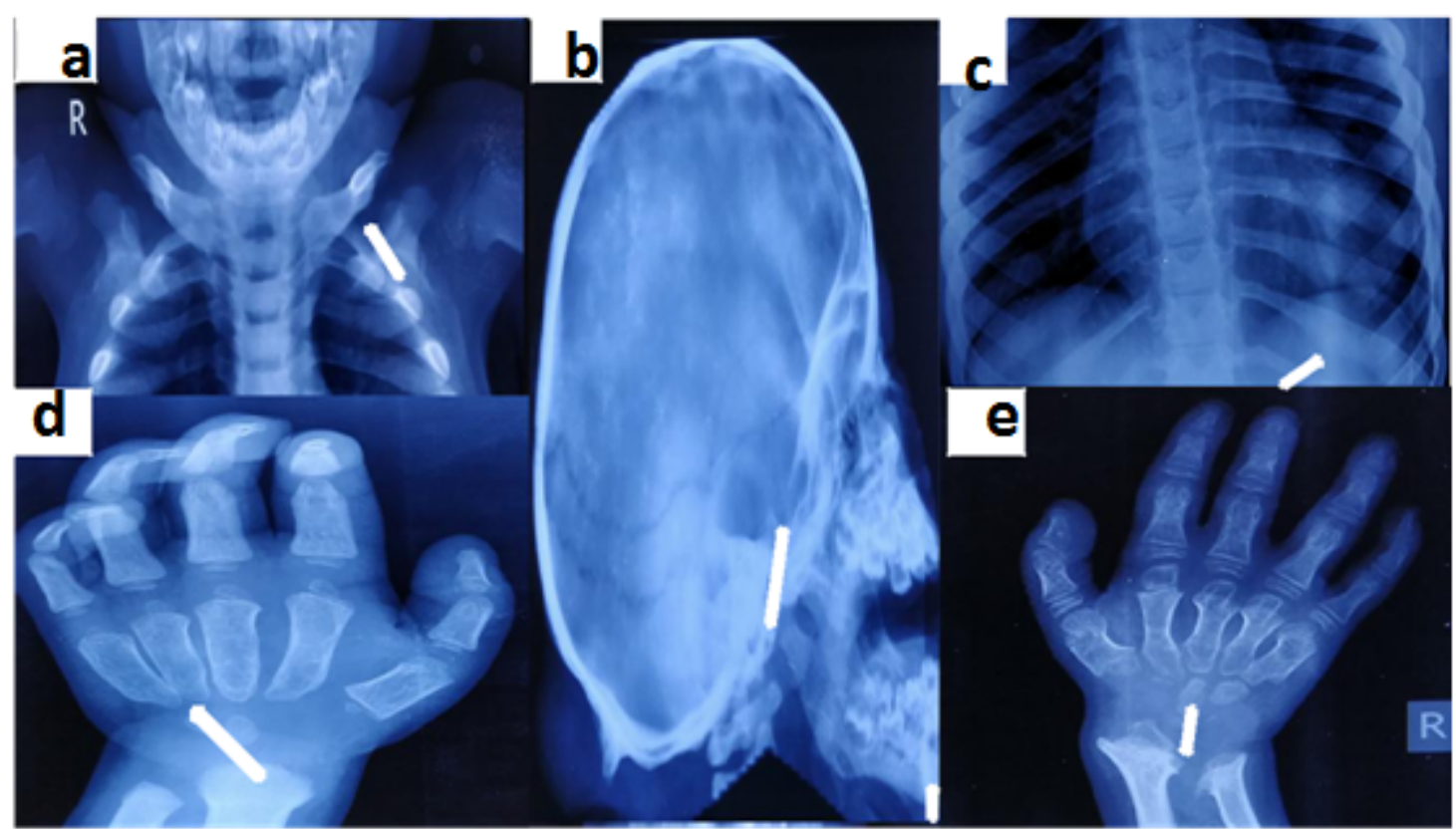

Figure 4. Dysostosis multiplex involving skull, ribs, clavicle and hands $\mathbf{A}$ - short and thick clavicles, B - J-shaped sella, C -Oar shaped ribs, D - Proximal tapering of metacarpals, $\mathbf{E}-\mathrm{V}$ shaped distal ulna and radius.

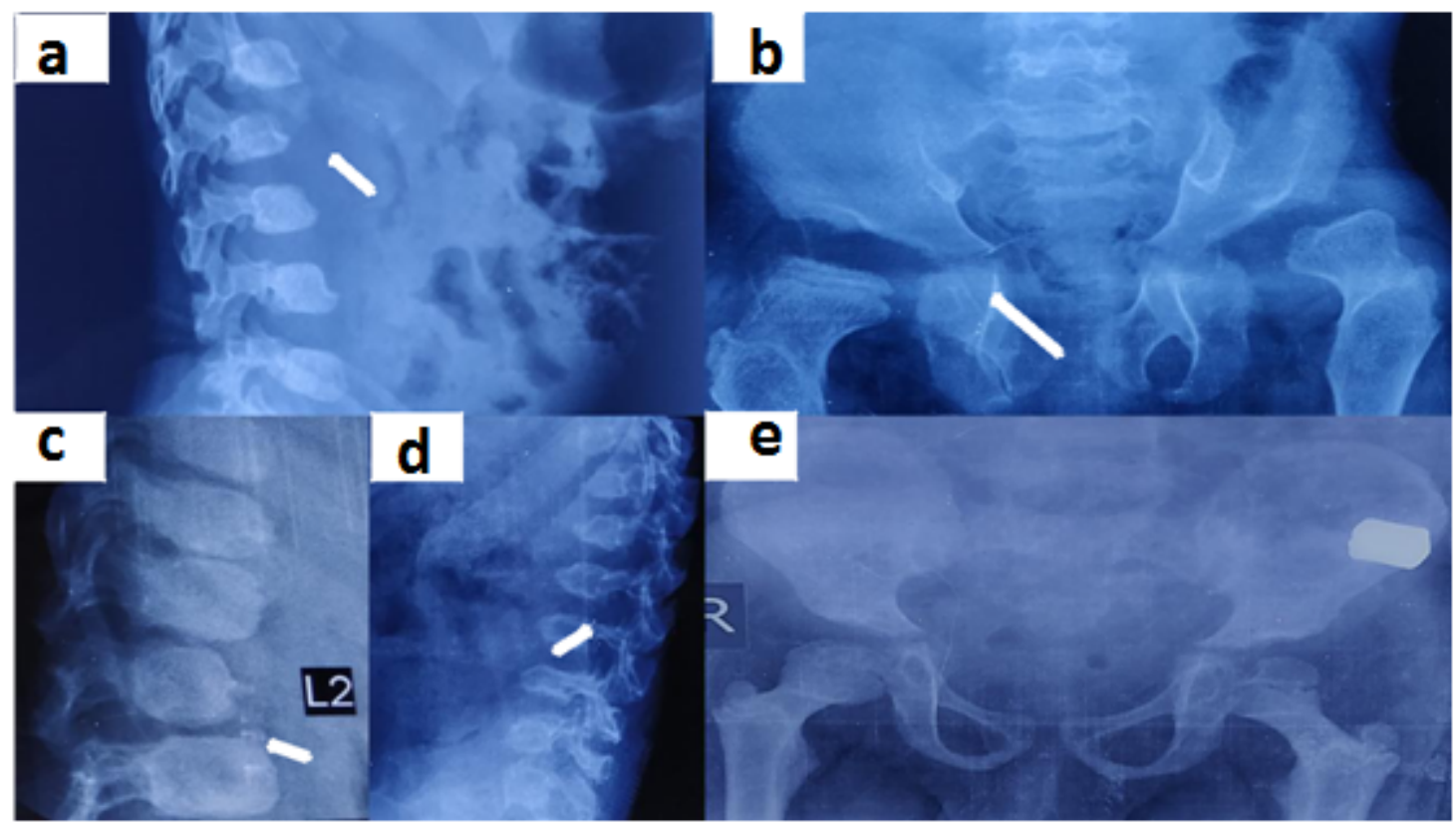

Figure 5. Dysostosis multiplex involving vertebral bod ies, pelvis and hip $\mathbf{5}^{\mathbf{a}}$ Inferior tapering of vertebral body, 5B - Poorly formed acetabulumhip dysplasia, 5C - Anterior hypoplasia of lumbar vertebra, 5D - Central beaking of vertebral body, 5E - Long femoral neck.

Robin spaces. Other rare findings were hydrocephalus, thinned out corpus callosum and colpocephaly.[6]

\section{Discussion}

Mucopolysaccharidosis is a group of common lysosomal storage disorders (LSDs) [1,2]. The commonest clinical features in MPS cases in present study were coarse facies, developmental delay, and short stature. Many children had all the above features at $1^{\text {st }}$ clinical visit and the median age at diagnosis was $4.5 y e a r s$ which indicates late presentation. Earlier, a family with 2 affected sibling with Hunter syndrome has been described from our centre, the older child had persistent thrombocytopenia.[5]

In present series, $41 \%$ MPS patients presented with recurrent respiratory problems. Thus, in children with recurrent respiratory problems, a high index of suspicion should be kept for MPS, and a skeletal survey should be performed. Dysostosis multiplex is the most common radiological finding in MPS [6]. This was present 
in all postnatal cases in present series and we ruled out non MPS cases based on negative skeletal survey. Combination of superior notching of vertebral body along with posterior scalloping is a very typical finding in MPS which is found in many of our subjects. Central beaking of vertebrae, and anterior hypoplasia of lumbar is found in Morquio syndrome.[7] Since many of them presented late they had irreversible complications by the time of diagnosis like intellectual disability and regression, short stature and contractures, valvulopathy etc. thus further affecting the quality of life. Though enzyme replacement therapy is available for selected types of MPS disorders, the therapy can be quite expensive and many families are unable to afford such therapy. Rehabilitation needs to be done in the fields of vision, hearing, musculoskeletal, development and neurological, pulmonary, cardiac etc to improve the quality of life.[8]

Hydrops is a prenatal manifestation in severe cases of lysosomal storage disorders, including MPS. So LSDs should be kept in the differential diagnosis of non-immune hydrops fetalis (NIHF) cases. Commoner causes of NIHF include cardiovascular abnormalities or lymphatic dysplasia in fetus apart from chromosomal anomalies.[9]

Knowledge of common manifestations like recurrent respiratory symptoms, contractures, and organomegaly can help in early diagnosis. Newborn screening program (NBS) is also one of the approaches for early diagnosis for lysosomal storage diseases including MPS even in the newborn period. [10] Countries like Philippines and Taiwan have started newborn screening for LSDs. Molecular identification or characterisation is possible by next generation sequencing (NGS) or Sanger sequencing. However variable clinical expression, the inability to predict phenotype, high cost of enzyme replacement therapy (ERT) and the lack of consensus about when to begin the treatment are issues to be considered especially in developing countries.

\section{Conclusions}

In our study, the mean age at diagnosis was 4.5 years and many have developed irreversible complications already at the $1^{\text {st }}$ clinical visit. This indicates that there is a need to increase the awareness about the slowly progressive symptoms of MPS in practicing paediatricians and other specialists. MPS should be suspected especially in patients with coarse facies, recurrent respiratory problems and short stature with or without contractures; which are common manifestations in MPS children. This may lead to an early diagnosis in these children. Diagnosis of co-morbidities is as important as diagnosing the disease itself because it will enable paediatricians to take appropriate measures for rehabilitation and plan appropriate management; all of which can help improve the quality of their life.

\section{Declaration of Conflicting Interests}

The authors declared no potential conflicts of interest with respect to the research, authorship, and/or publication of this article.

\section{References}

1. Caciotti A, Garman SC, Rivera-Colón Y, et al. GM1 gangliosidosis and Morquio B disease: An update on genetic alterations and clinical findings. Biochim Biophys Acta. 2011;1812(7):782-790. doi: 10.1016/j.bbadis.2011.03.018

2. Robert K, Bonita S, St.Geme, Schor. Nelson Textbook of Pediatrics. 20th ed. Elsevier; 2016.

3. Khan SA, Peracha H, Ballhausen D, et al. Epidemiology of mucopolysaccharidoses. Mol Genet Metab. 2017;121(3):227240. doi: 10.1016/j.ymgme.2017.05.016

4. Chiong MAD, Canson DM, Abacan MAR, Baluyot MMP, Cordero CP, Silao CLT. Clinical, biochemical and molecular characteristics of Filipino patients with mucopolysaccharidosis type II - Hunter syndrome. Orphanet J Rare Dis. 2017;12:7. doi: 10.1186/s13023016-0558-0

5. Panigrahi I, Dhanorkar M, Didel S, Koganti RA. Hunter syndrome with persistent thrombocytopenia. BMJ Case Rep. 2019;12(4):e226518. doi: 10.1136/bcr-2018-226518

6. Lanza ML, Attinà G, Palmucci S, Mauro LA, Belfiore G, Barone R, Fiumara A, Sorge G, Ettorre GC. Imaging findings of mucopolysaccharidoses. Poster presented at: European Society of Radiology's, ECR 2012. 2012; doi: 10.1594/ecr2012/C-2180

7. Lachman RS, Burton BK, Clarke LA, et al. Mucopolysaccharidosis IVA (Morquio A syndrome) and VI (Maroteaux-Lamy syndrome): under-recognized and challenging to diagnose. Skeletal Radiol. 2014;43(3):359369. doi: 10.1007/s00256-013-1797-y

8. Lampe C, McNelly B, Gevorkian AK, et al. Transition of patients with mucopolysaccharidosis from paediatric to adult care. Mol Genet Metab Rep. 2019;21:100508. doi: 10.1016/j.ymgmr.2019.100508

9. Bellini C, Donarini G, Paladini D, Calevo MG, Bellini T, Ramenghi LA, Hennekam RC. Etiology of nonimmune hydrops fetalis: An update. Am J Med Genet A. 2015;167A(5):1082-1088. doi: 10.1002/ajmg.a.36988

10. Donati MA, Pasquini E, Spada M, Polo G, Burlina A. Newborn screening in mucopolysaccharidoses. Ital J Pediatr. 2018;44(suppl 2):126. doi: 10.1186/s13052-0180552-3 